Bryn Mawr College

Scholarship, Research, and Creative Work at Bryn Mawr

College

2007

\title{
The Breakdown of the Fingerprinting of Vortices by Hysteresis Loops in Circular Multilayer Ring Arrays
}

V. Rose

Xuemei Cheng

Bryn Mawr College, xcheng@brynmawr.edu

D. J. Keavney

J.W. Freeland

K.S. Buchanan

See next page for additional authors

Let us know how access to this document benefits you.

Follow this and additional works at: http://repository.brynmawr.edu/physics_pubs

Part of the Physics Commons

\section{Custom Citation}

V. Rose et al., Appl. Phys. Lett. 91, 132501 (2007).

This paper is posted at Scholarship, Research, and Creative Work at Bryn Mawr College. http://repository.brynmawr.edu/physics_pubs/63 
Authors

V. Rose, Xuemei Cheng, D. J. Keavney, J. W. Freeland, K. S. Buchanan, B. Ilic, and V. Metlushko 


\title{
The breakdown of the fingerprinting of vortices by hysteresis loops in circular multilayer ring arrays
}

\author{
V. Rose, ${ }^{a}$ X. M. Cheng, D. J. Keavney, and J. W. Freeland \\ Advanced Photon Source, Argonne National Laboratory, Argonne, Illinois 60439, USA \\ K. S. Buchanan \\ Center for Nanoscale Materials, Argonne National Laboratory, Argonne, Illinois 60439, USA \\ B. Ilic \\ Cornell Nanofabrication Facility, School of Applied and Engineering Physics, Cornell University, Ithaca, \\ New York 14853, USA \\ V. Metlushko \\ Electrical and Computer Engineering, University of Illinois at Chicago, Chicago, Illinois 60607, USA
}

(Received 20 July 2007; accepted 9 August 2007; published online 24 September 2007)

\begin{abstract}
Microscale single-layer ferromagnetic rings typically exhibit a magnetic vortex state at remanence, characterized by a flux-closed magnetic state with zero stray fields. Magnetic reversal in such systems yields a vanishing remanent magnetization. In contrast, the authors show that in individual layers in thin rings, which alternate magnetic and nonmagnetic materials $(\mathrm{NiFe} / \mathrm{Cu} / \mathrm{Co})$, layer-resolved hysteresis loops, measured using x-ray resonant magnetic scattering, exhibit the characteristics of a vortex formation, although photoelectron emission microscopy and micromagnetic simulations clearly prove that multidomain states are formed. This result is of considerable importance for the development of pseudo-spin-valve-type structures for applications. (C) 2007 American Institute of Physics. [DOI: 10.1063/1.2786856]
\end{abstract}

The study of magnetism of multilayered structures has revealed many properties with considerable technological potential, especially for advanced magnetic-based sensors and magnetic random-access memories (MRAMs). ${ }^{1,2}$ Conventionally, a MRAM consists of two ferromagnetic (FM) layers separated by a thin isolating tunneling barrier. The electrical tunneling transport across the insulating barrier is spin dependent and is controlled by the relative orientation of the magnetization in the two FM layers. Another, in principle, different but extremely promising design for the realization of a MRAM device is a multilayer stack of alternating hard and soft FM rings that are separated by a nonmagnetic $\mathrm{Cu}$ ring. ${ }^{3}$ This pseudo-spin-valve design utilizes the vertical giant magnetoresistive effect and takes advantage of magnetic vortex states with controlled chirality within each FM ring. A magnetic vortex state is characterized by a flux-closure magnetization with zero stray fields. ${ }^{4}$ Because the flux-closed vortex state reduces interactions between neighboring elements, this concept may enable devices with high-density elements and robust and repeatable switching characteristics. However, recent investigations of $\mathrm{NiFe} / \mathrm{Cu} / \mathrm{Co}$ dot arrays show that vortex formation in multilayers may be suppressed due to dipolar field contributions. ${ }^{5}$ In addition, depending on the geometrical ring parameters, such as thickness, width, and outer diameter, the spin switching in FM rings may vary. ${ }^{6}$ In thin singlelayer rings, e.g., switching can occur directly from a bidomain state, also referred to as an onion state, into the reverse onion state, leaving out the nucleation of a magnetic vortex state. ${ }^{7}$ In the onion state, the magnetization follows the circumference of the ring with opposite senses of circulation in

\footnotetext{
${ }^{\text {a) }}$ Author to whom correspondence should be addressed. Electronic mail: vrose@anl.gov
}

the two-halves of the ring. Thus, two opposite head-to-head domain walls are formed generating a stray field.

While the experimental study of planar arrangements of several FM rings has attracted attention because of their fascinating properties, ${ }^{8-10}$ in practice, characterizing magnetic multilayers is challenging. Nevertheless, a rich magnetic phase diagram has recently been proposed for nanorings consisting of alternate layers of magnetic and nonmagnetic materials. ${ }^{11}$ We show, however, that due to interlayer dipolar coupling, the interpretation of magnetic reversals cannot be easily transferred from the established knowledge in singlelayer rings to pseudo-spin-valve-type structures.

Arrays of circular polycrystalline Co (thickness of $5 \mathrm{~nm}$ ) and $\mathrm{NiFe}(5 \mathrm{~nm})$ single layers and $\mathrm{NiFe}(5 \mathrm{~nm}) / \mathrm{Cu}$ $(3 \mathrm{~nm}) / \mathrm{Co}(5 \mathrm{~nm})$ multilayer rings have been fabricated on $\mathrm{Si}$ wafers by means of electron-beam lithography on a double-layered positive-type resist, followed by electronbeam evaporation and a subsequent ultrasonic assisted chemical lift-off process. Each ring had a width of $0.75 \mu \mathrm{m}$ and an outer diameter of $2 \mu \mathrm{m}$ [Fig. 1(a)]. In order to prevent the magnetic layers from oxidation, a 3-nm-thick $\mathrm{Cu}$ film was deposited on top of each sample.

Since the structures to be investigated involve multilayer systems, it is necessary to go beyond macroscopic magnetization measurements and separate out the magnetic signals of the individual magnetic layers. For that reason, x-ray resonant magnetic scattering (XRMS) and photoelectron emission microscopy (PEEM) measurements were performed at beamline 4ID-C of the Advanced Photon Source at Argonne National Laboratory. ${ }^{12}$ Furthermore, to gain further insight into the measurements, we have performed micromagnetic simulations using the LLG Micromagnetics Simulator software. ${ }^{13}$ The scattering geometry is illustrated in Fig. 1(b). The field $H$ was parallel to the sample surface and in the 


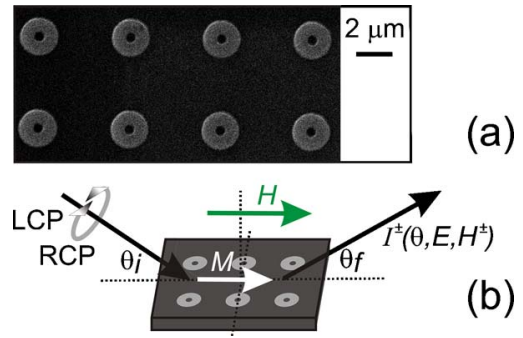

FIG. 1. (Color online) (a) Scanning electron micrograph of rings with a width of $0.75 \mu \mathrm{m}$ and an outer diameter of $2 \mu \mathrm{m}$. (b) X-ray resonant magnetic scattering setup. Left and right circularly polarized (LCP and RCP) x-rays probe the magnetization $M$ along the applied field $H$. At specular conditions $\left(\theta_{i}=\theta_{f}\right)$, reflected intensities $I^{ \pm}$are measured as a function of incident energies $E$ and $H$.

scattering plane. Using circular polarized $\mathrm{x}$ rays, the laterally averaged magnetization $M$ component parallel to the field was probed. For the micromagnetic simulations, each layer was represented using a two-dimensional mesh with a cell size of $12 \times 12 \mathrm{~nm}^{2}$. The parameters of $\mathrm{NiFe}(\mathrm{Co})$ used in the simulations are the exchange constant $C=2 A=2.1$ $\times 10^{-6} \mathrm{erg} / \mathrm{cm}\left(6.1 \times 10^{-6} \mathrm{erg} / \mathrm{cm}\right)$, and the saturation magnetization $M_{s}=800 \mathrm{emu} / \mathrm{cm}^{3}\left(1414 \mathrm{emu} / \mathrm{cm}^{3}\right)$, resulting in an exchange length of $R_{0}=C^{1 / 2} / M_{s}=18.1 \mathrm{~nm}(17.5 \mathrm{~nm})$. Crystalline anisotropy was neglected for the Permalloy, and for the Co a uniaxial anisotropy of $4 \times 10^{6} \mathrm{erg} / \mathrm{cm}^{3}$ was used with an out-of-plane easy axis. In the multilayers, only dipolar interactions were considered because interlayer exchange should be negligible for the spacer thicknesses used in the experiments.

In Fig. 2, we present the normalized field-dependent XRMS signal of $\mathrm{Co}$ and $\mathrm{NiFe}$ single-layered ring arrays as well as the reversal in the $\mathrm{Co}$ and $\mathrm{NiFe}$ layers of the pseudospin-valve structure. The experimental data are compared to the results of micromagnetic simulations. In the Co singlelayer ring, the coercivity $H_{c}$ amounts to $32 \mathrm{Oe}$ and the remanent magnetization $M_{r}$ is 0.97 of the saturation value $M_{s}$ [Fig. 2(a)]. The NiFe ring qualitatively shows a different, more round shape [Fig. 2(b)]. Here, $H_{c}$ is only 18 Oe and $M_{r}=0.46 M_{s}$. While for the Co ring the simulation is in good agreement with the experimental data, the theory overestimates the coercivity in the NiFe ring. In both single-layer rings, the reversal occurs with a single transition between onion states. At the switching field, the spins coherently rotate into the antiparallel configuration. ${ }^{14}$ This switching characteristic is typical for thin $\mathrm{FM}$ rings. ${ }^{4}$ In the $\mathrm{NiFe} / \mathrm{Cu} / \mathrm{Co}$ multilayer ring array, the reversal characteristics and the switching fields for the soft and hard layers differ considerably from those of the isolated single-layer rings [Figs. 2(c) and 2(d)]. In the Co layer [Fig. 2(c)], the coercivity $H_{c}$ increases to 56 Oe. As compared to the single-layer ring, the hysteresis loop shows a reduction of $M_{r}$ to $0.77 M_{s}$. The micromagnetic simulations qualitatively confirm this trend of increasing $H_{c}$ with decreasing $M_{r}$. Likewise, a good qualitative agreement between experiment and simulation is found in the reversal of the NiFe layer [Fig. 2(d)]. The reversal exhibits a reduced coercivity compared to the single-layer ring. Lowering the field from positive saturation [open symbols in Fig. 2(d)], $M_{r}$ almost vanishes when $H$ approaches zero and is already negative in the simulation. This predicts a switching in the NiFe ring even before the field is reversed, indicating a strong dipolar interaction between the FM lay-
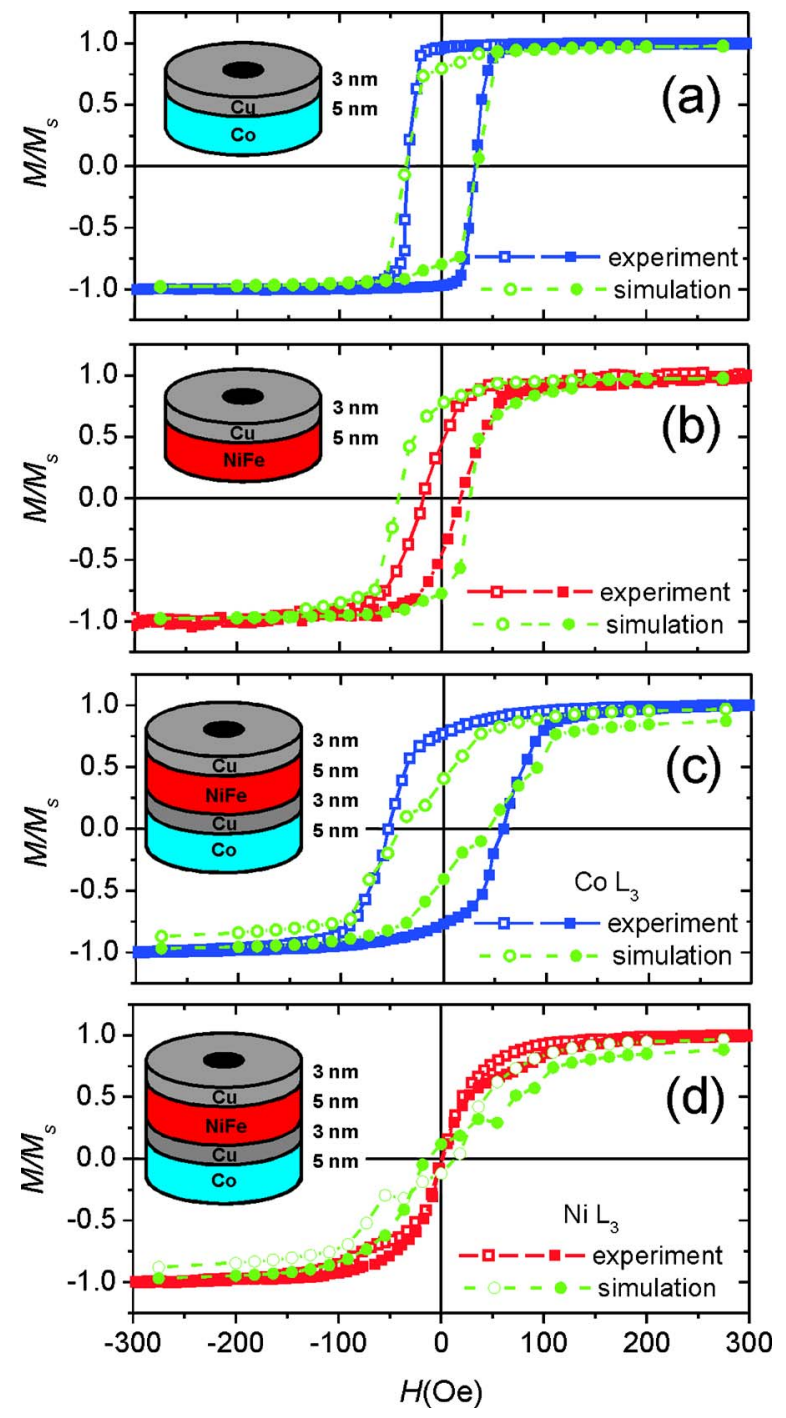

FIG. 2. (Color online) Normalized XRMS signal as a function of applied field of (a) Co rings ( $5 \mathrm{~nm}$ thick), (b) NiFe rings $(5 \mathrm{~nm}$ ), (c) the Co layer of a $\mathrm{NiFe}(5 \mathrm{~nm}) / \mathrm{Cu}(3 \mathrm{~nm}) / \mathrm{Co}(5 \mathrm{~nm})$ ring structure, and (d) the NiFe layer of a $\mathrm{NiFe} / \mathrm{Cu} / \mathrm{Co}$ structure. All samples are capped with $3 \mathrm{~nm} \mathrm{Cu}$ to prevent oxidation. Open symbols illustrate the magnetization path for declining external field, and full symbols indicate the opposite direction. Experimental data (squares) are compared to micromagnetic simulations (circles).

ers. Then, for negative $H$, the sign of the curvature of the loop changes, giving rise to a plateau in both the experimental data and the simulation. Generally, a low remanence and the appearance of pronounced steps are a notable feature of hysteresis loops. In the case of isolated single-layer rings, a small $M_{r}$ is the consequence of a vortex formation, and steps are assigned to the movement of a magnetic vortex core in the ring perimeter with subsequent annihilation. ${ }^{4}$ Remarkably, even though the loops show this characteristic fingerprint of a vortex formation, it does not correspond to vortex formation in these multilayer rings as described below.

Figure 3 shows the magnetic domain structure in the $\mathrm{NiFe}$ and Co layers of the pseudo-spin-valve ring structure derived from the micromagnetic simulations. At remanence, antivortex-type states are formed. While in quadrants I and III, the spins predominantly point in the direction of the previously applied field, the magnetization in II and IV is perpendicular with respect to the field. In the Co layer, the contribution of $M$ in quadrants II and IV cancels each other out, reducing $M_{r}$ to approximately $\frac{1}{2} M_{s}$ [Fig. 2(c)]. Likewise, 


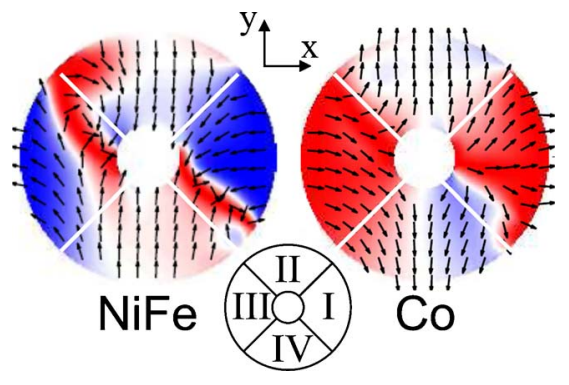

FIG. 3. (Color online) Micromagnetic simulations of the spin configuration in the NiFe (left) and Co (right) layers in the pseudo-spin-valve-type ring structure at remanence. Antivortex states are formed, where the direction of the magnetization preferentially is pointing inward (I, II, and IV in NiFe; III in $\mathrm{Co}$ ) and outward (III in NiFe; I, II, and IV in Co) to the rings. The external field was applied in the $x$ direction.

quadrants II and IV of the NiFe ring yield a low $M_{r}$. Nevertheless, the spin distribution in quadrants I and III of the magnetically soft $\mathrm{NiFe}$ layer is not as uniform, having a small remanence value. Despite this fact, the majority of the spins are already switched into the opposite field direction, which causes the negative $M_{r}$ [Fig. 2(d)]. Because the $\mathrm{Cu}$ spacer thickness is small, the interlayer dipolar coupling becomes significant.

The formation of multidomain states in the pseudo-spinvalve structure is also conclusively demonstrated using PEEM magnetic domain imaging measurements. In Fig. 4, the $\mathrm{x}$-ray energy was tuned to the $\mathrm{Ni} L_{3}$ edge. The PEEM image displays an obviously nonuniform domain structure, with different rings showing various multidomain states. A comparison with the results of the micromagnetic simulation for the NiFe layer shows that less than $5 \%$ of the rings can be attributed to the predicted antivortex-type state. This sug-

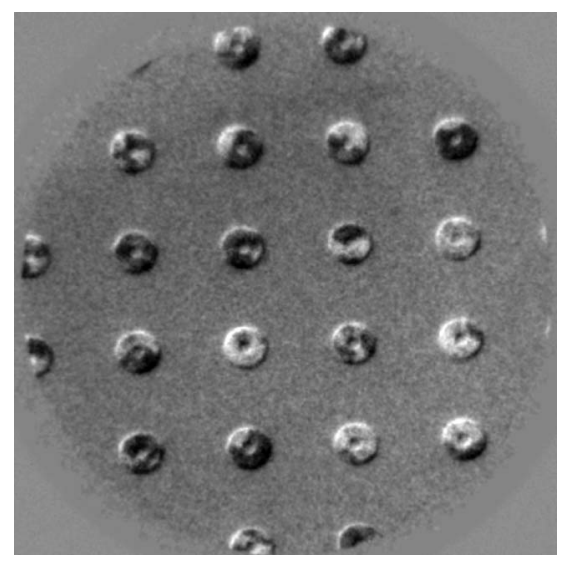

FIG. 4. PEEM image of the NiFe layer of the multilayer rings exhibits diverse multi-domain structures at remanence. This suggests that imperfections in the rings strongly control the magnetic reversal. gests that slight structural imperfections and rough layer boundaries cause ring specific magnetic switching behavior. The individual details of each ring may significantly influence the nucleation, location, and mobility of domain walls. The measurements also indicate that there may be multiple reversal paths that have only a small difference in energy. An analysis of the black-to-white ratio in 147 different multilayer rings yields an $M_{r} / M_{s}$ value of around $0.2 M_{s}$. This ratio is almost certainly an overestimation of the remanence, since it is not clear that any region of the sample is fully magnetized along the $x$ direction. It does show, however, that the remanence value is very small as seen in the XRMS measurements and in the micromagnetic simulations.

In conclusion, the reversal of FM layers in multilayer ring structures can significantly differ from those of its constituents. In particular, multidomain states such as antivortex states can be stabilized in $\mathrm{NiFe}$ or Co rings when they are part of a $\mathrm{NiFe} / \mathrm{Cu} / \mathrm{Co}$ heterostructure. In particular, care has to be taken in the interpretation of hysteresis loops of multilayers; the characteristics of single-layer hysteresis loops might not be transferable. This property is very important in the design of magnetic devices using pseudo-spin-valve elements.

This work was supported by the U. S. Department of Energy, Office of Science, Office of Basic Energy Sciences, Contract No. DE-AC02-06CH11357, and by the National Science Foundation, Grant No. ECS-0202780 (V.M.).

${ }^{1}$ E. Hirota, H. Sakakima, and K. Inomata, Giant Magneto-Resistance Devices (Springer, Berlin, 2002), Vol. 40, p. 135.

${ }^{2} \mathrm{U}$. Hartmann and R. Coehoorn, Magnetic Multilayers and Giant Magnetoresistance: Fundamentals and Industrial Applications (Springer, Berlin, 2000), Vol. 37, p. 49.

${ }^{3}$ J.-G. Zhu, Y. Zheng, and G. A. Prinz, J. Appl. Phys. 87, 6668 (2000).

${ }^{4}$ M. Kläui, C. A. F. Vaz, L. Lopez-Diaz, and J. A. C. Bland, J. Phys.: Condens. Matter 15, R985 (2003).

${ }^{5}$ Y. Choi, D. R. Lee, J. W. Freeland, G. Srajer, and V. Metlushko, Appl. Phys. Lett. 88, 112502 (2006).

${ }^{6}$ S. P. Li, D. Peyrade, M. Natali, A. Lebib, Y. Chen, U. Ebels, L. D. Buda, and K. Ounadjela, Phys. Rev. Lett. 86, 1102 (2001).

${ }^{7}$ M. Kläui, Adv. Solid State Phys. 44, 479 (2004).

${ }^{8}$ V. Rose, K. Buchanan, S.-H. Chung, M. Grimsditch, V. Metlushko, A. Hoffmann, V. Novosad, S. D. Bader, and H. Ibach, Phys. Rev. B 73, 094442 (2006).

${ }^{9}$ U. Welp, V. K. Vlasko-Vlasov, G. W. Crabtree, J. Hiller, N. Zaluzec, V. Metlushko, and B. Ilic, J. Appl. Phys. 93, 7056 (2003).

${ }^{10}$ V. Novosad, K. Y. Guslienko, H. Shima, Y. Otani, S. G. Kim, K. Fukamichi, N. Kikuchi, O. Kitakami, and Y. Shimada, Phys. Rev. B 65, 060402 (2002).

${ }^{11}$ J. Escrig, P. Landeros, D. Altbir, M. Bahiana, and J. d'Albuquerque e Castro, Appl. Phys. Lett. 89, 132501 (2006).

${ }^{12}$ J. W. Freeland, J. C. Lang, G. Srajer, R. W. Winarski, D. Shu, and D. M. Mills, Rev. Sci. Instrum. 73, 1408 (2002).

${ }^{13}$ M. R. Scheinfein and E. A. Price, LLG Micromagnetics Simulator, 2003.

${ }^{14}$ N. Dao and S. L. Whittenburg, IEEE Trans. Magn. 39, 2525 (2003). 